\title{
Characterization of EMI filters based on metamaterials
}

\author{
I. Gil ${ }^{(1)}$, R. Fernández ${ }^{(1)}$, Y. Vives ${ }^{(2)}$, R. Jauregui $^{(2)}$ and F. Silva ${ }^{(2)}$ \\ Departament d'Enginyeria Electrònica (DEE), Universitat Politècnica de Catalunya (UPC) \\ (1) 08222 Colom 1, Terrassa (Barcelona), SPAIN \\ ${ }^{(2)} 08034$ Jordi Girona 1-3, Barcelona, SPAIN \\ Email: gilgali@eel.upc.edu
}

\begin{abstract}
EMI filters based on metamaterials. The filters are developed by means of sub-wavelength resonators and designed to have notch-type attenuation in the $2.45 \mathrm{GHz}$ band. Two types of filters based on SRR and CSRR rings are presented. The simulated responses by MoM and FDTD are compared with the measurement data obtained from the developed prototypes.
\end{abstract}

Keywords: EMI filters, microstrip, metamaterials.

\section{INTRODUCTION}

The increasing of the electromagnetic emissions in the last years involves the design of novel electronic systems which are immune to electromagnetic interferences (EMI). Specifically, the recent and rapid growth of wireless communication systems has increased the demand of immunity to radiofrequency interference (RFI) design techniques in order to achieve the EMC. Several approaches have been presented in order to reduce cost and dimensions of conventional EMI filters such as the design of optimized circuits in order to obtain a high-immunity to RFI [1] or the implementation of Electromagnetic Band Gaps (i.e. metamaterials based on periodic structures with period in the range of the wavelength) with the aim to reduce the EMI in printed circuit boards (PCB) [2].

Moreover, in recent years several filter applications at the frequency range of microwaves have been developed by means of metamaterials based on sub-wavelength resonators such as the split-ring resonator (SRR) [3] or the complementary split-ring resonator (CSRR) [4].

This work has been partially supported by the Spanish Ministerio de Educación y Ciencia under project DPI2007-63878.
In this work, the characterization by numerical simulation (MoM and FDTD) and the measurement of these particles in order to develop microstrip EMI filters centred at $2.45 \mathrm{GHz}$ is discussed for applications at the industrial, scientific and medical band (ISM).

\section{SYNTHESIS OF THE EMI FILTERS}

SRRs consist of a pair of metal rings etched on a dielectric slab with apertures in opposite sides (Figure 1) and are mainly excited by means of an external magnetic flux along the ring's axis. The key aspect of such resonators is the fact that they are electrically small and exhibit an effective permeability which is negative in a narrow band above their resonant frequency. Therefore, SRRs are able to inhibit signal propagation in the vicinity of the resonance frequency which satisfies the condition $\mu<0$. Since these resonators present dimensions significantly smaller than signal wavelength at their resonance frequency, they allow reducing circuit area. Furthermore, a significant cost reduction is achieved, since no lumped elements are needed in order to perform the EMI filter.

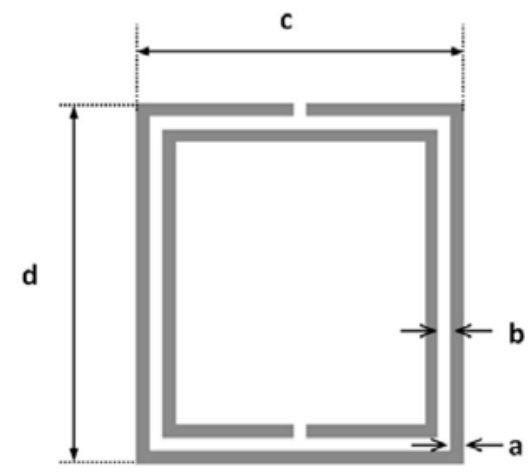

Figure 1. Topology of the square shaped SRR with its relevant dimensions. 
Alternatively to the SRRs, notch filters can be also implemented by means of their dual counterparts, the complementary split-ring resonators (CSRRs, see Figure 2). These resonators are the negative image of SRRs and present a behaviour consisting of $\varepsilon<0$. Therefore, by etching CSRRs in the ground plane underneath of a microstrip line (victim line) it is possible to reject EMI signals which frequency corresponds to the particles resonance frequency. This effect is basically carried out by means of a significant electrical coupling between the host victim line and the resonators.

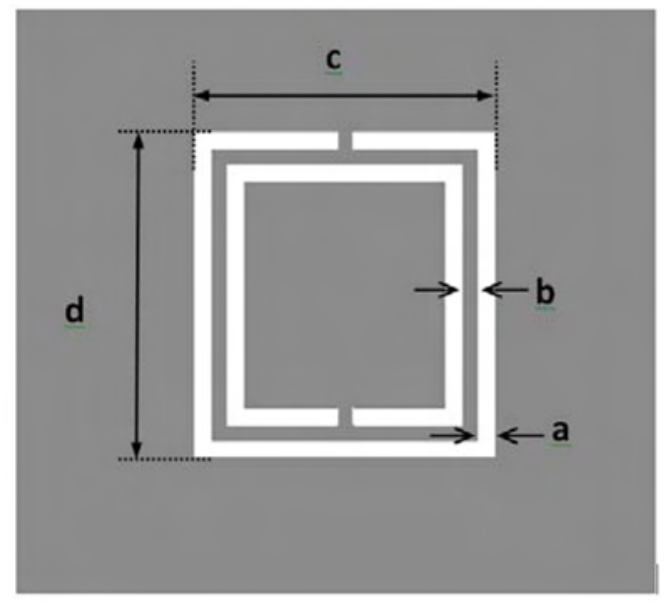

Figure 2. Topology of the square shaped CSRR with its relevant dimensions. Grey zone corresponds to ground plane metal.

The first proposed EMI filter is depicted in Figure 3. It consists of 4-stage SRRs magnetically coupled, at their resonance frequency, to a victim microstrip transmission line. The dimensions of the SRRs are slightly modified in several stages in order to achieve, slightly different resonance frequency operation and, thus, a wider stopband response.

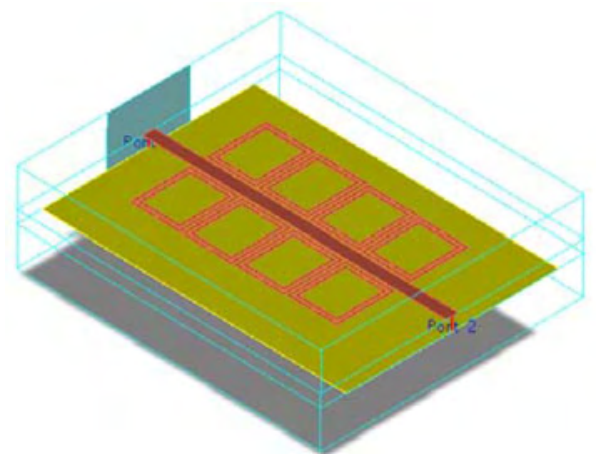

Figure 3. Layout of the characterized 4-stage EMI SRR filter Dimensions are: host victim line: width $=1.18 \mathrm{~mm}$, length $=30.6 \mathrm{~mm}$; SRRs: $a=b=200 \mu \mathrm{m} ; \quad \mathrm{c}=5000 \mu \mathrm{m} ; 1,2$-stage $\mathrm{d}=6000 \mu \mathrm{m} ; 3$-stage $\mathrm{d}=5900 \mu \mathrm{m}$; 4-stage $\mathrm{d}=5800 \mu \mathrm{m}$.
A second 5-stage EMI CSRR filter has been designed and characterized (Figure 4). The main advantage of CSRRs is their virtual non-area consumption, since they are etched in the ground plane. Therefore, from the point of view of the designer the top metal layer remains unaltered. Also they add no extra cost to the implementation, since CSRRs are etched by using a conventional drilling machine.

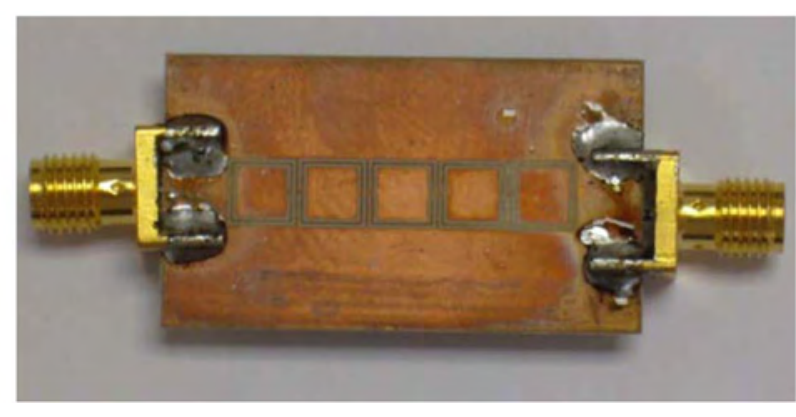

Figure 4. Test prototype of the characterized 5-stage EMI CSRR filter. Dimensions are: host victim line: $w i d t h=1.18 \mathrm{~mm}$, length $=35.5 \mathrm{~mm}$; CSRRs: $a=b=200 \mu \mathrm{m} ; c=5000 \mu \mathrm{m} ; 1$-stage $d=5400 \mu \mathrm{m}, 2,3,4-$ stage $d=5200 \mu \mathrm{m} ; 5$-stage $d=5000 \mu \mathrm{m}$

The frequency operation of both prototypes has been designed in the same frequency band (ISM, $f_{0}=2.45 \mathrm{GHz}$ ). Both EMI filters have been designed by using a Rogers RO3010 substrate (which parameters are: $\varepsilon_{r}=10.2$ and thickness, $h=1.27 \mathrm{~mm}$ ). In the design process a $50 \Omega$ microstrip line has been designed by means of Agilent Linecalc calculator.

The experimental results of both EMI filter shown in the following Figures have been measured by means of a Rohde \& Schwarz ZVRE vector network analyzer.

\section{Simulation Of THE EMI FILTERS}

The electromagnetic simulations have been performed by using two methods: the Method of Moments (MoM) and the Finite-Difference TimeDomain (FDTD) method by means of Agilent Momentum and SEMCAD 13.4, respectively.

Concerning the MoM method, a $5 \mathrm{GHz}$ mesh frequency together with a 20cells/wavelength mesh density has been used in both cases. The minimum size of mesh used in FDTD method is of $0.01 \mathrm{~mm}$ and 1.5 MCells. A Pentium Core ${ }^{\mathrm{TM}} 2$ Duo $2.66 \mathrm{~GB}$ processor and 8-Gbyte RAM have been used for the calculation. 


\section{RESUlts}

Figures $5 \mathrm{a}$ and $5 \mathrm{~b}$ show the EMI SRR filter simulations results in comparison with the experimental data. As can be observed, the momentum method fits accurately the resonance frequency area, whereas the FDTD method achieves a good matching concerning the band pass frequency range, in terms of losses. In fact, the results reveal an excellent agreement regarding simulations. A clear matching with the frequency of the rejected bands is achieved.
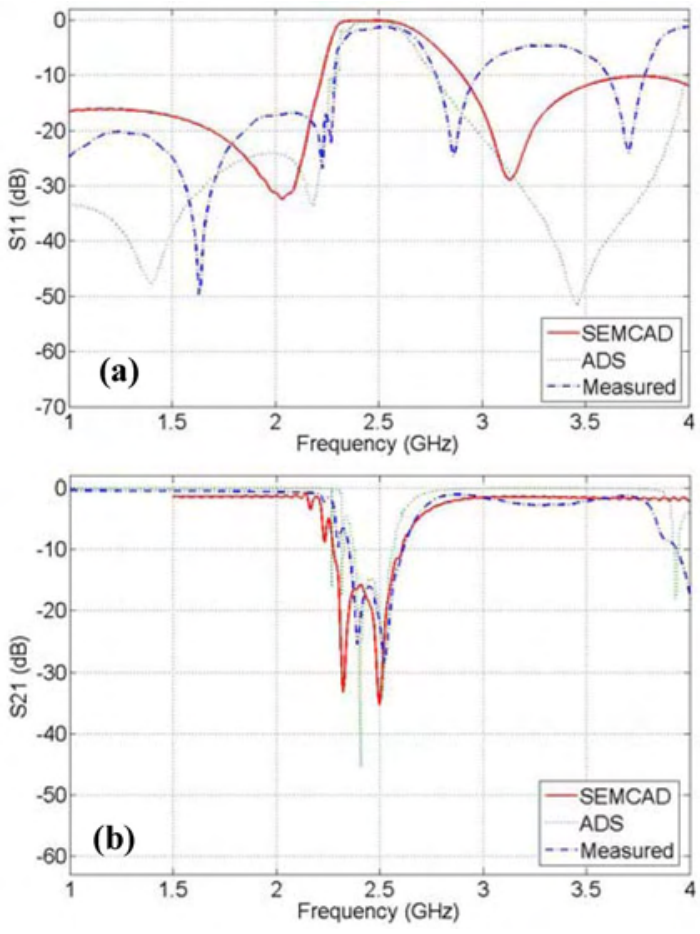

Figure 5. SRR filter measured and simulated results (a) return losses, S11, (b) insertion losses, S21

Figures $6 \mathrm{a}$ and $6 \mathrm{~b}$ depict the simulation and experimental results of the return and insertion losses of the EMI CSRR filter. As can be seen, we can also achieve a notable matching between simulations and experimental in MoM simulations. FDTD simulations show a good agreement with the measurements providing smoother responses in S11.

Concerning the rejection level, in case of EMI SRR filter the measured values correspond to $20 \mathrm{~dB}$ for the frequency of interest, whereas the EMI CSRR filters present a refection level in the order of $40 \mathrm{~dB}$, due to the higher number of stages. Both experimental fractional bandwidth correspond to $10 \%$.
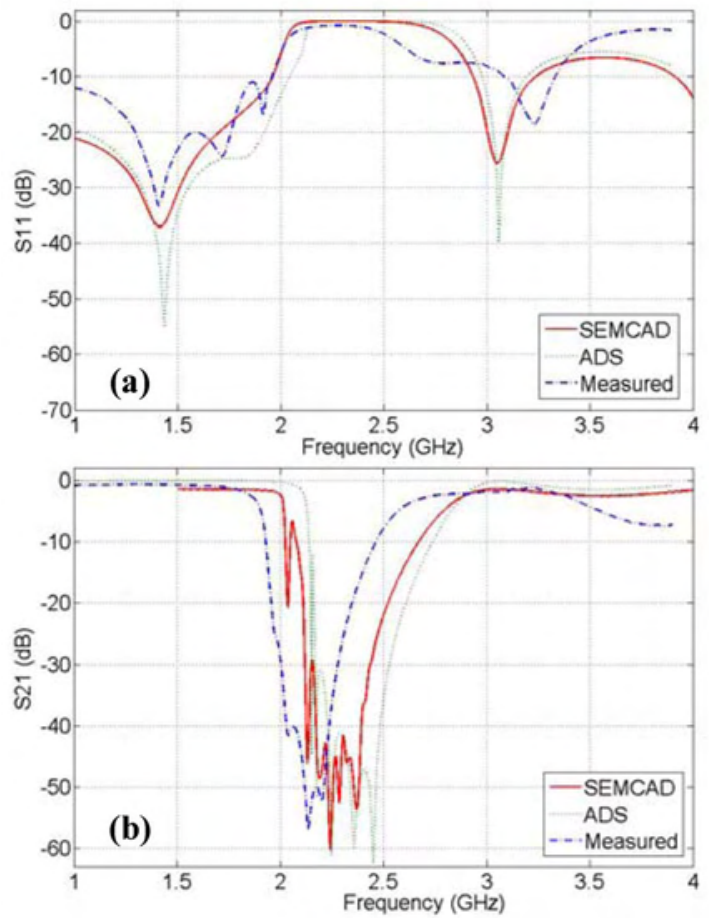

Figure 6. CSRR filter measured and simulated results (a) return losses, S11, (b) insertion losses, S21

\section{CONCLUSIONS}

In this work, two approaches for the design of EMI filters at ISM band have been considered by using the electrical small resonators SRR and CSRR. The prototypes have been simulated and analyzed by performing two different simulation process the characterization by numerical simulation (MoM and FDTD). The experimental results match notably with both simulation methods.

\section{REFERENCES}

[1] F. Fiori and P.S. Crovetti, "Complementary differential pair with high immunity to RFI", Electronics Letters, Vol. 38, pp. 1663-1664, December 2002.

[2] S. Shahparnia and O.M. Ramahi, "Electromagnetic interference (EMI) reduction from printed circuit boards (PCB) using electromagnetic bandgap structure", IEEE Transactions on Electromagnetic Compatibility, Vol. 46, pp. 580-587, November 2004.

[3] J.B. Pendry, A.J. Holden, D.J. Robbins and W.J. Stewart, "Magnetism from conductors and enhanced nonlinear phenomena", IEEE Trans. Microwave Theory Tech., 47, 2075 (1999). 